\title{
IdeAs
}

Idées d'Amériques

1| 2011

Intégrations dans les Amériques

\section{Integración en las Américas: nuevas perspectivas socioeconómicas}

Intégrations dans les Amériques : nouvelles perspectives socio-économiques

New socio-economic perspectives on integration in the Americas

\section{Christine Zumello y Jean-Baptiste Velut}

Traductor: Marta Gómez

\section{(2) OpenEdition \\ 1 Journals}

Edición electrónica

URL: https://journals.openedition.org/ideas/3037

DOI: $10.4000 /$ ideas.3037

ISSN: 1950-5701

Este artículo es una traducción de:

Intégrations dans les Amériques: Nouvelles perspectives socio-économiques - URL : https://

journals.openedition.org/ideas/103 [fr]

Otras traducciones del artículo:

New socio-economic perspectives on integration in the Americas - URL : https://

journals.openedition.org/ideas/3031 [en]

Integrações nas Américas: Novas perspectivas socioeconômicas - URL : https://

journals.openedition.org/ideas/3043 [pt]

Editor

Institut des Amériques

Referencia electrónica

Christine Zumello y Jean-Baptiste Velut, «Integración en las Américas: nuevas perspectivas socioeconómicas», IdeAs [En línea], 1 | 2011, Publicado el 01 septiembre 2011, consultado el 20 octubre 2022. URL: http://journals.openedition.org/ideas/3037 ; DOI: https://doi.org/10.4000/ideas. 3037

Este documento fue generado automáticamente el 20 octubre 2022.

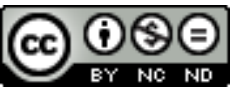

Creative Commons - Atribución-NoComercial-SinDerivadas 4.0 Internacional - CC BY-NC-ND 4.0 https://creativecommons.org/licenses/by-nc-nd/4.0/ 


\title{
Integración en las Américas: nuevas perspectivas socioeconómicas
}

\author{
Intégrations dans les Amériques : nouvelles perspectives socio-économiques \\ New socio-economic perspectives on integration in the Americas
}

Christine Zumello y Jean-Baptiste Velut

Tradución : Marta Gómez

1 La última década del siglo XX ha estado marcada por una aceleración de las dinámicas de integración regional en todo el mundo. En la mayoría de los casos, el regionalismo se ha impuesto como una respuesta estratégica a la intensificación de la competencia internacional en una economía cada vez más globalizada. El continente americano ha sido escenario de multitud de proyectos de integración regional declinados bajo formas diversas en función de su alcance geográfico (bilateralismo/multilateralismo), su orientación ideológica (neoliberalismo, alternativa bolivariana, etc.), sus relaciones de poder (Norte-Sur, Sur-Sur), su grado de integración (acuerdo sectorial, mercado común, zona de libre comercio) y, de modo más general, de la «profundidad» de los acuerdos regionales, esto es, de su carácter vinculante y del poder decisorio de las instituciones supranacionales.

2 Si bien este polimorfismo del (de los) regionalismo(s) en las Américas excluye cualquier conclusión apresurada sobre el costo y los beneficios de la integración regional, sí permite plantear la cuestión de los desafíos y las modalidades socioeconómicas de dichos procesos. ¿La integración económica regional se limita principalmente a la fusión de las esferas productivas y financieras? ¿Qué cuestiones políticas se consideran prioritarias? ¿Qué ámbitos se excluyen, formal u oficiosamente, de las dinámicas regionalistas?

3 En relación con las modalidades del regionalismo, se trata de examinar la estructura y el papel de las instituciones regionales, que constituyen la encarnación más tangible de la voluntad política dada a un proyecto de integración. Así, el grado de aplicación de un acuerdo regional, el funcionamiento y el poder real de los organismos supranacionales, o el presupuesto asignado a un proyecto, son a menudo indicios del vigor de los 
fenómenos de integración. Asimismo, la naturaleza democrática del proceso decisorio $\mathrm{y}$, en particular, la estructura jerárquica y la dimensión participativa de un proyecto, tienen con frecuencia un impacto directo sobre su legitimidad y, consecuentemente, sobre su durabilidad.

Más allá de estas modalidades institucionales, los regionalismos también se construyen fuera de los caminos trillados de la diplomacia. A la integración oficial se opone la integración «real», sustentada en los flujos migratorios, los intercambios comerciales (lícitos e ilícitos) y las inversiones. Las convergencias y divergencias entre estas dos formas de integración plantean, también en este caso, numerosas cuestiones relacionadas con la democracia, el desarrollo o la seguridad. ¿Cuáles dinámicas formales e informales estructuran el regionalismo? ¿Cabe hablar de distintos modelos de integración dentro del continente americano que sintetizarían las complejas relaciones entre Estados, mercados, instituciones regionales y ciudadanos, del mismo modo que los «modelos de capitalismo» ${ }^{1}$ han permitido esquematizar la economía política de los Estados, por ejemplo? De estas modalidades derivan las consecuencias económicas y sociales de las políticas regionalistas, fruto del capital político invertido por los arquitectos de la integración o resultados ineluctables o imprevistos de los procesos impulsados.

5 En este primer número de la revista IdeAs nos proponemos analizar los desafíos y las modalidades socioeconómicas de la integración regional en las Américas a través de diversas experiencias regionales, entre ellas, el TLCAN, el Tratado de libre comercio entre Estados Unidos y Centroamérica (CAFTA, por sus siglas en inglés) o el Sistema de la Integración Centroamericana (SICA). El presente dossier reúne los trabajos de investigadores norteamericanos, franceses y latinoamericanos, que ofrecen miradas interdisciplinares sobre las características intrínsecas del regionalismo en las Américas. El objetivo es, por un lado, establecer un primer balance socioeconómico de estas dos últimas décadas de integración hasta la crisis financiera -cuya propagación en el hemisferio occidental está directamente relacionada con la interdependencia cada vez mayor de las economías del continente- y, por otro lado, proponer un análisis prospectivo de los principales desafíos que deberán afrontar los futuros arquitectos de las políticas regionalistas. ¿La globalización de las cadenas de producción convierte en obsoleto el modelo regionalista? ¿Qué soluciones deberán proponer los gobiernos del continente americano para corregir las deficiencias socioeconómicas de los regionalismos? ¿El futuro de la integración regional pasará por el declive, la ampliación o la profundización de estos procesos?

El dossier inicia con un artículo de Sherrie Baver sobre los desafíos medioambientales de la integración regional en Norteamérica y, más concretamente, sobre la relación entre el libre comercio y la protección medioambiental a través de la experiencia del TLCAN. Desde un enfoque institucionalista, la autora analiza el impacto del dispositivo institucional del TLCAN en la puesta en marcha de reformas medioambientales en México. Baver examina el precedente histórico creado por las instituciones medioambientales norteamericanas y su capacidad para reorientar a largo plazo el modelo de libre comercio americano hacia una vía más ecológica.

7 Josette Altmann Borbón adopta un enfoque más global de los fenómenos de integración en Centroamérica y analiza los desafíos y los límites del regionalismo, así como su capacidad de resistencia frente a la globalización. La autora hace balance de los procesos de regionalización de las tres últimas décadas y pone de relieve los desafíos 
sociales y políticos a los que deberán enfrentarse los países centroamericanos para satisfacer las aspiraciones democráticas y de desarrollo de sus pueblos. Entre las paradojas y las deficiencias o insuficiencias del regionalismo centroamericano, la autora analiza los conflictos entre los diferentes modelos de integración (Norte-Sur, Sur-Sur) dentro de la región, la falta de voluntad política de los encargados de tomar decisiones, así como la fragilidad de las instituciones regionales.

Helen Chang, por su parte, compara las experiencias de integración norteamericana y centroamericana a través de un análisis econométrico de los efectos del libre comercio en la sincronización de los ciclos económicos. El objetivo es doble. En primer lugar, se trata de evaluar la dependencia de las economías latinoamericanas con respecto a Estados Unidos. En efecto, si bien la sincronización macroeconómica tiende a reducir la volatilidad de los países en vías de desarrollo, también puede incrementar su inestabilidad en periodos de crisis debido a los efectos de arrastre (spillover effects). En una segunda parte, la autora estudia las soluciones que deben adoptarse frente a este dilema midiendo el grado de coordinación de las políticas monetarias y presupuestarias entre los miembros del TLCAN, por un lado, y del CAFTA, por otro.

Jean-Baptiste Velut combina igualmente diagnóstico y prescripción a través de un estudio sobre los efectos del TLCAN en la economía mexicana quince años después de la entrada en vigor del tratado. En un análisis de la evolución de los flujos comerciales, de la inversión y de los indicadores sociales mexicanos, el autor detalla las imperfecciones del modelo regional norteamericano. Velut muestra las paradojas de la integración norteamericana $\mathrm{y}$, en particular, los conflictos entre, por un lado, las modalidades institucionales y la lógica estrictamente productiva del TLCAN, y por otro, la realidad social de la integración norteamericana y los límites del tratado en dicha cuestión.

Estas tensiones se abordan también en el trabajo de Fanny Lauby, que analiza las tendencias de la inmigración mexicana hacia Estados Unidos en el periodo post-TLCAN. En contra de las expectativas de los responsables políticos norteamericanos, el TLCAN no ha sido capaz de frenar los flujos de población hacia el Norte. Este fracaso se debe no solo a la ausencia de cláusulas migratorias en el acuerdo sino también a la militarización de la frontera entre México y Estados Unidos, que se ha convertido en un fenómeno de perdurabilidad de la inmigración clandestina. La autora conceptualiza la emergencia de un tipo de regionalismo informal alimentado por los flujos de inmigración hacia el Norte y por las transferencias de fondos de los migrantes hacia el Sur. Lauby analiza las características de este sistema paralelo al proceso institucional del TLCAN, así como sus retos para la alianza Estados Unidos-México.

11 Este compendio de trabajos, producto de tradiciones universitarias diversas, ilustra las fortalezas y debilidades de los procesos de integración regional. Asimismo, abre nuevas perspectivas y plantea numerosos interrogantes sobre el futuro de los regionalismos en las Américas, cuyos logros futuros pasarán indiscutiblemente por una redefinición de las modalidades de integración que también tome en consideración los imperativos sociales y medioambientales del continente americano. 


\section{NOTAS}

1. Peter A. Hall \& David Soskice (eds.), Varieties of Capitalism: the Institutional Foundations of Comparative Advantage, Oxford: OUP, 2001.

\section{AUTORES}

\section{CHRISTINE ZUMELLO}

Christine Zumello est maître de conférences en civilisation américaine à l'Université de la Sorbonne Nouvelle-Paris 3. Elle travaille plus particulièrement sur les institutions politiques américaines et les questions financières. Elle est membre du CERVEPAS, Centre de Recherche sur la Vie Economique dans les Pays Anglo-Saxons (EA 3960).

\section{JEAN-BAPTISTE VELUT}

Jean-Baptiste Velut est maître de Conférences en Civilisation Américaine à l'Université Sorbonne Nouvelle - Paris III. jean-baptiste.velut@univ-paris3.fr 